\section{P191 WHO IS PROVIDING HIV TESTING AND DIAGNOSIS? COMPARING GENERAL PRACTITIONERS AND SEXUAL HEALTH CENTRES IN FIVE REGIONS IN THE NETHERLANDS}

1,2,3 D Twisk*, ${ }^{4} S$ Bogers* ${ }^{*}{ }^{5} \mathrm{~L}$ Beckers, ${ }^{1,2,3} \mathrm{H}$ Götz, ${ }^{1,3} \mathrm{~A}$ Meima, ${ }^{7} \mathrm{M}$ Kroone, ${ }^{7} \mathrm{E}$ Hoornenborg, ${ }^{8} \mathrm{~A}$ Ott, ${ }^{9} \mathrm{M}$ Luning-Koster, ${ }^{10,11} \mathrm{~N}$ Dukers-Muijrers, ${ }^{11,12} \mathrm{C}$ Hoebe, ${ }^{13} \mathrm{C}$ Kampman, ${ }^{14} \mathrm{~F}$ Bosma, ${ }^{1,7} \mathrm{M}$ Schim van der Loeff, ${ }^{4} \mathrm{~S}$ Geerlings, ${ }^{5,6,15} \mathrm{~J}$ van Bergen. ${ }^{1}$ Department of Infectious Disease Control, Municipal Public Health Service RotterdamRijnmond, Rotterdam, The Netherlands; 'Department of Public Health, Erasmus MC, University Medical Center Rotterdam, Rotterdam, The Netherlands; ${ }^{3}$ Department Research and Business Intelligence, Municipality of Rotterdam, Rotterdam, The Netherlands; ${ }^{4}$ Department of Internal Medicine, Division of Infectious Diseases, Amsterdam University Medical Centers, location Academic Medical Center, Amsterdam, The Netherlands; ${ }^{5}$ Department of General Practice, Amsterdam University Medical Centers, location Academic Medical Center, Amsterdam, The Netherlands; ${ }^{6}$ Centre for Infectious Disease Control, National Institute for Public Health and the Environment (RIVM), Bilthoven, The Netherlands; 'Department of Infectious Diseases, Public Health Service Amsterdam, Amsterdam, The Netherlands; ${ }^{8}$ Certe, Department of Medical Microbiology, Groningen, The Netherlands; ${ }^{9} G G D$ Fryslan, Municipal Public Health Service Leeuwarden, Leeuwarden, The Netherlands; ${ }^{10}$ Department of Health Promotion, Care and Public Health Research Institute (CAPHRI), Maastricht, The Netherlands; ${ }^{11}$ Department of Sexual Health, Infectious Diseases and Environmental Health, South Limburg Public Health Service, Heerlen, The Netherlands; ${ }^{12}$ Department of Social Medicine and Medical Microbiology, Care and Public Health Research Institute (CAPHRI), Maastricht University Medical Centre (MUMC+), Maastricht, The Netherlands; ${ }^{13}$ Public Health Service Twente, Enschede, The Netherlands; ${ }^{14}$ Laboratory for Medical Microbiology and Public Health, Hengelo, The Netherlands; ${ }^{15}$ STI AIDS Netherlands (SOA AIDS Nederland), Amsterdam, The Netherlands

\subsection{6/sextrans-2021-sti.282}

Background General practitioners (GPs) and sexual health centres (SHCs) are the main providers of STI and HIV testing in the Netherlands. We compared HIV testing by GPs versus SHCs to gain insight in strategies to improve HIV testing, stimulating timely HIV diagnosis.

Methods Laboratory data (2011-2018) on HIV testing by GPs and SHCs in five Dutch regions with varying levels of urbanisation were used. Mean regional HIV testing rates per 10,000 residents $\geq 15$ years were compared between providers using negative binomial generalised additive models, and additionally stratified by sex and age $(15-29 y, 30-44 y, 45-59 y$, $\geq 60 \mathrm{y})$. Chi-squared tests were used to compare percentage positive between providers.

Results Analysed data included 507,197 HIV tests (GP 36\%, SHC 64\%). The highest HIV testing rates and positivity were observed in highly urbanised regions, with large variation between regions. The HIV testing rates ranged from 28-178/ 10,000 residents by GPs and from 30-379/10,000 residents by SHCs. Testing rates by GPs were lower than by SHCs in two regions, while these rates were comparable in the others. In all regions, men were tested less by GPs than by SHCs; for women this varied per region. Among 15-29 year olds, GPs' testing rates were lower than SHCs', while this was reversed in older age categories. The overall mean HIV positivity was $0.5 \%$ for GPs and $0.4 \%$ for SHCs. In Amsterdam, positivity was higher among individuals tested by GPs, but in the other regions no difference was observed.

Conclusion This is the first study comparing HIV testing by GPs versus SHCs using laboratory data in the Netherlands. Our results show that besides SHCs, GPs also play a key role, especially in some subgroups, but large regional variation exists. Regional-specific interventions to improve GPs' HIV testing practices are needed for populations not attending the SHCs to ensure timely HIV diagnosis.

\section{P192 NEVER TESTED FOR HIV: DIRECTIONS FOR TARGETED TESTING INTERVENTIONS AMONG MEN WHO HAVE SEX WITH MEN}

${ }^{1} \mathrm{H}$ Zimmermann ${ }^{*},{ }^{1} \mathrm{~W}$ van Bilsen, ${ }^{1,2} \mathrm{~A}$ Boyd, ${ }^{1,3} \mathrm{U}$ Davidovich, on behalf of HIV Transmission Elimination Initiative Amsterdam (H-TEAM). ${ }^{1}$ Public Health Service Amsterdam, Amsterdam, The Netherlands; ${ }^{2} H I V$ Monitoring Foundation, Amsterdam, The Netherlands; ${ }^{3}$ Department of Social Psychology, University of Amsterdam, Amsterdam, The Netherlands

\subsection{6/sextrans-2021-sti.283}

Men who have sex with men (MSM) who are unaware of their HIV-infection contribute to onward HIV transmission and are more likely to progress to symptomatic HIV disease. We assessed determinants of never-testing for HIV among MSM living in the Netherlands. Between April-July 2019, we conducted a nationwide cross-sectional survey among MSM on socio-demographics, HIV-testing behavior and sexual risk taking, which was distributed via gay networking sites/apps. Multivariable logistic regression was used to investigate characteristics associated with never-testing for HIV.

950 HIV-negative tested and 122 never-tested MSM completed the survey. In never-tested MSM, median age was 37 $(\mathrm{IQR}=22-51)$ years and 37 (30\%) reported recent sexual risk behavior. Never-testing was associated with younger age (adjusted odds ratio [aOR] per year increase $=0.98$, 95\%-confidence interval $[\mathrm{CI}]=0.97-1.00, \mathrm{p}=0.021$ ), having sex with men and women $(\mathrm{aOR}=2.93,95 \%-\mathrm{CI}=1.61-5.34, \mathrm{p}<0.001)$, and not knowing others living with $\mathrm{HIV}(\mathrm{aOR}=3.74$, 95\%$\mathrm{CI}=2.28-6.13, \mathrm{p}<0.001)$. A significant interaction effect between education level and residential area was observed $(p=0.001)$. Among higher-educated MSM, those living outside a large urban area had higher odds of never-testing compared to those living in an urban area $(\mathrm{aOR}=6.26,95 \%$ $\mathrm{CI}=2.42-16.24, \mathrm{p}<0.001)$. Lower-educated MSM had higher odds of never-testing irrespective of residential area (urban: $\mathrm{aOR}=12.06,95 \% \mathrm{CI}=4.00-36.38$; rural: aOR 9.29, 95\% $\mathrm{CI}=3.64-23.76 ; \mathrm{p}<0.001$ for both). Among MSM with recent sexual risk, never-testing was associated with having sex with men and women $(\mathrm{aOR}=2.80,95 \%-\mathrm{CI}=1.09-7.18$, $\mathrm{p}=0.032)$ and not knowing others with HIV $(\mathrm{aOR}=4.91$, $95 \%-\mathrm{CI}=1.97-12.24, \mathrm{p}=0.001)$. Based on this online sample of MSM, never-testing for HIV is more common in those who were younger, had lower education level, non-urban residency, had sex with men and women, and did not know someone with HIV. Only the latter two factors were observed among those with recent sexual risk behavior. Testing interventions for those never-tested should be tailored to residential area and education level, and inclusive of bisexuality.

\section{P193 BACTERICIDAL ACTIVITY OF ESCULETIN IS ASSOCIATED WITH IMPAIRED CELL WALL SYNTHESIS BY TARGETING GLUTAMATE RACEMASE OF NEISSERIA GONORRHOEAE}

'D Saluja*, ${ }^{1} \mathrm{~A}$ Pawar, ${ }^{1} \mathrm{C}$ Konwar, ${ }^{2} \mathrm{U}$ Chaudhry, ${ }^{1} \mathrm{M}$ Chopra, ${ }^{1} \mathrm{P}$ Jha. ${ }^{1}$ University of Delhi, Delhi, India; ${ }^{2}$ Bhaskaracharya College of Applied Sciences, Delhi, India

10.1136/sextrans-2021-sti.284

Background Antimicrobial resistance in Neisseria gonorrhoeae (NG), the causative organism of gonorrhea, has posed a 
serious threat worldwide. Gonorrhea is a sexually transmitted disease with a high morbidity burden and is an important cause of pelvic inflammatory disease. The failure of recommended dual therapy with ceftriaxone and azithromycin has compromised the general and reproductive health of infected individuals. Thereby, Neisseria gonorrhoeae was recently classified as a 'Priority 2' microorganism by the World Health Organization. Consequently, persistent attempts are under way to discover novel drug targets as well as new drugs to fight against Neisseria. In this direction, considerable number of phytochemicals have been reconnoitred for their remedial intercession via targeting bacterial proteins.

Methods MurI gene is specific to the bacterial kingdom, it can be exploited as a potential drug target for the treatment of bacterial diseases. Accordingly, diverse families of phytochemicals were screened in silico for their binding affinity with NG-MurI protein. Esculetin, one of the shortlisted compounds, was evaluated for its functional, structural and antibacterial activity. MurI was cloned, expressed and purified to homogeneity and used for testing the effect of esculetin on its racemase activity under invitro conditions. We further evaluated the effect of esculetin on sensitive and drug resistant strains of NG.

Results We screened various classes of natural compounds and found esculetin, a coumarin derivative as a potent compound to target its effect on the growth of Neisseria gonorrhoeae. Treatment with esculetin resulted in growth inhibition, cell wall damage and altered permeability as revealed by fluorescence and electron microscopy. Furthermore, esculetin inhibited racemization activity of recombinant, purified MurI protein of NG, an important enzyme required for peptidoglycan biosynthesis.

Conclusions Our results suggest that esculetin could be further explored as a lead compound for developing new drug molecules against multidrug resistant strains.

\section{P194 PARTICIPATORY COMMUNITY-BASED MAPPING OF PEOPLE WHO INJECT DRUGS (PWID) LOCATIONS TO IMPROVE HIV CASE-FINDING}

${ }^{1} \mathrm{O}$ Kovtun*, ${ }^{2} \mathrm{M}$ Malakhova, ${ }^{1} \mathrm{~T}$ Saliuk. ${ }^{1}$ The International Charitable Foundation 'Alliance for Public Health', Kyiv, Ukraine; ${ }^{2}$ Alliance Consultancy, Kyiv, Ukraine

\subsection{6/sextrans-2021-sti.285}

Background HIV in Ukraine continues to be concentrated among PWID. According to the IBBS, only 58\% of HIV-positive PWID are aware of their status, so more than 33.000 PWID living with HIV remain undiagnosed. The mapping of PWID locations makes it possible to optimize the HIV casefinding activities, which include both stationary testing sites and outreach routes.

Methods We used community-based participatory research (CBPR) approach to mapping the locations of PWID in 12 cities. The study was conducted in collaboration with leading community-based organizations. We identified the locations in each city, the approximate number of PWID in its, and the schedule for visiting each location (peak days and hours). Data on current locations of HIV testing sites and outreach routes were used for additional analysis.

Results 885 PWID locations of 24 different types were identified (from 31 to 223 in each city). Among them are pharmacies, streets/yards/garages, shops, markets, places of drug sale, parks, places of PWID accumulation, pawnshops/banks, hospitals, transport stops, cafes, entertainment points, railway stations, probation, rehabilitation centers, social and psychological assistance points. $63.3 \%$ of locations are not associated with medical and social services and were previously unknown to HIV-services. Locations were visited all days of the week from $6.00 \mathrm{am}$ to $0.05 \mathrm{am}$.

The mapping results were visualized using Google Maps and QGIS and compared with HIV-projects data. The current location of the sites and outreach routes does not allow the full reach of PWID and relies on the most famous gathering places.

Conclusions Data on the PWID locations are often incompletely known to HIV testing services, which limits the successful HIV cases-finding among them. Experience with community-based mapping has made it possible to re-plan routes and relocate fixed sites to better reach PWID. Obtaining such comprehensive results would not be impossible without collaboration with PWID-community.

\section{P195 SEXUALIZED DRUG USE AMONG FEMALE SEX WORKERS FROM EIGHT CITIES IN CHINA}

${ }^{1} \mathrm{M}$ Xiong*, ${ }^{2} \mathrm{~J}$ Ong, ${ }^{1} \mathrm{Y}$ Wang, ${ }^{1} \mathrm{~W}$ Tang, ${ }^{1} \mathrm{C}$ Wang. ${ }^{1}$ Dermatology Hospital of Southern Medical University, Guangzhou, China; 'Central Clinical School, Monash University, Melbourne, Australia

\subsection{6/sextrans-2021-sti.286}

Background There is rich literature on sexualized drug use (i. e. drug use before or during sex) for men who have sex with men but less data from female sex workers (FSW) particularly from low- and middle-income countries. We describe the sociodemographic characteristics, sexual behaviors and HIV and sexually transmitted infection (STI) testing behaviors among FSW reporting sexualized drug use, compared with FSW who do not report sexualized drug use.

Methods This cross-sectional study was conducted in eight cities in China in 2019. We recruited FSW through community organizations working with sex workers. Multivariable logistic regression models were used to evaluate the factors associated with FSW reporting sexualized drug use. We adjusted for current injecting drug use, age, marital status, migration status, income and education in each of the models.

Results In total, 1287 women participated: average age was $35 \cdot 1$ years (SD 10.3), about half reported a monthly income over 5000 RMB (\$USD 707, 52.4\%), and a minority completed high school or above (17.4\%). Among participants, 284 $(22 \cdot 1 \%$, 95\% CI:19.8-24.4) reported a history of sexualized drug use. Compared to FSW who never reported a history of sexualized drug use or IDU, FSW who reported a history of sexualized drug use had greater odds of: having a manager (adjusted odds ratio (AOR) 2.10, 95\% CI:1.47-2.99), reporting inconsistent condom use for vaginal sex (AOR 2.67, 95\% CI:1.93-3.69), inconsistent condom use for oral sex (AOR 2.33, 95\% CI:1.25-4.37), ever had an unintended pregnancy (AOR 1.43, 95\% CI:1.04-1.96), and ever diagnosed with STIs (AOR 5·39, 95\% CI:3·92-7·40).

Conclusion We recommend routinely asking FSW about sexualized drug use as nearly one in five FSW reported a history of sexualized drug use and these women had an elevated risk profile compared with those who reported no sexualized drug use. 